\title{
Late recurrences of Sydenham's chorea are not associated with anti-basal ganglia antibodies
}

\author{
N A Harrison, A Church, A Nisbet, P Rudge, G Giovannoni
}

J Neurol Neurosurg Psychiatry 2004;75:1478-1479. doi: 10.1136/jnnp.2003.030775

Anti-basal ganglia antibodies ( $A B G A$ ) have been associated with $100 \%$ of acute cases and $69 \%$ of persistent cases of Sydenham's chorea. We describe two cases of late recurrences of Sydenham's chorea with absence of ABGA. Both patients had several childhood episodes of Sydenham's chorea. MRI imaging of the basal ganglia and exhaustive investigations for other causes of chorea were normal or negative. The absence of ABGA may be evidence against an autoimmune pathology in late and some persistent recurrences. We suggest the likely pathophysiology to be dopamine hypersensitivity of chronically damaged basal ganglia neurones possibly following induction of an autoimmune antibody response in childhood.

$\mathrm{S}$ ydenham's chorea (SC) is a delayed complication of group A $\beta$-haemolytic streptococcal infection typically, although not exclusively, associated with acute rheumatic fever. Approximately a quarter of patients with rheumatic fever develop SC within 6 months of the acute streptococcal infection. ${ }^{1}$ Rheumatic fever remains a common and serious disease in the developing world and its incidence and that of SC is increasing again in the developed world. ${ }^{2}$

Clinically SC is characterised by chorea with or without other motor symptoms including: facial grimacing, hypotonia, loss of fine motor control, and gait disturbance. ${ }^{3}$ Neuropsychiatric symptoms are also commonly seen. ${ }^{45}$ Half of the acute cases resolve within 2-6 months ${ }^{3}$ with mild or moderate chorea persisting in $50 \%$ of patients for 2 years or more. ${ }^{1}$

Pathological and imaging studies have implicated the basal ganglia as the seat of the pathology in SC. In the 1920s Greenfield and Wolfsohn showed neuronal loss within the basal ganglia with an associated leucocytic infiltrate and evidence of plasma cells. ${ }^{6}$ More recently, volumetric magnetic resonance imaging (MRI) studies have shown enlargement of the basal ganglia with no changes seen in other brain areas. $^{7}$ Clinical improvement following the use of dopamine antagonists also points to a basal ganglia pathology.

In 1976 Husby et al identified an autoantibody that reacted to a constituent of subthalamic and caudate neurones in a proportion of patients with SC. ${ }^{8}$ Further studies have shown that this anti-basal ganglia antibody (ABGA) is present in the serum in up to $100 \%$ of cases of acute SC and $69 \%$ of cases of persistent SC. ${ }^{9}$ The target of ABGA is currently unknown so the effects on striatal neurones are unclear. Case studies of patients with SC treated with immunomodulating drugs such as intravenous immunoglobulin and steroids have reported an improvement in symptoms and provide evidence that ABGA may be pathogenic in SC.

We describe two patients who presented with chorea in their 70s. Both had SC in their youth and, in particular, had a prolonged course or recurrences in their teenage years or early 20s. Thorough investigation for other causes of chorea proved negative. We suggest that these patients have late recurrences of SC possibly precipitated by neuronal loss associated with the ageing process. In contrast to cases of acute or early recurrences of SC, both these cases were serum ABGA negative. We suggest that unlike acute relapses of SC, late recurrences are unlikely to have an immune pathophysiological basis and hypothesise that they are more likely to be a consequence of dopamine hypersensitivity in chronically damaged basal ganglia.

\section{CASE REPORTS}

\section{Case 1}

A 77 year old female retired shop assistant was admitted with a 3 year history of chorea, personality change, and cognitive impairment. At 12 years of age she had developed rheumatic fever and then SC, which persisted for 2 years. Following this she made a full recovery. She was well until the age of 74 years when her husband noticed a change in her personality with increasing memory difficulties. She developed bizarre behaviour, exhibiting obsessional traits such as accumulating used tea bags and filling empty plastic milk bottles with water. She developed involuntary twitching movements of the face that progressed to involve the neck and then her limbs, which progressed to chorea. Genetic testing for Huntington's and DRPLA expansions and the SCA 3 mutation were all negative. Acanthocytes were absent and treponema haemagglutination was negative. Copper studies, antistreptolysin O titre, ESR, thyroid function tests and all autoantibodies including anti-phospholipid antibodies were normal or negative. CSF showed a mildly raised protein of $0.86 \mathrm{~g} / \mathrm{l}$, five lymphocytesand a normal glucose ratio. Oligoclonal bands were negative in both CSF and serum. MRI of the brain showed diffuse cortical atrophy. ABGA as determined by indirect immunofluorescence, ELISA, and western immunoblot were negative.

\section{Case 2}

A patient in the eighth decade presented with a 2 year history of progressive choreiform movements. The patient had developed St Vitus dance at the age of 7 years with a recurrence at 14 and was hospitalised for 2 years. The patient remained well but following a minor accident developed chorea, predominantly of the right foot. The chorea gradually spread to involve the whole body.

TPHA, antistreptolysin O titre, ESR, anti-cardiolipin antibodies, full blood count, and electrolytes were all normal. Thyroid stimulating hormone was slightly raised but free T3 and T4 were within the normal range. An MRI scan showed marked cerebral and cerebellar atrophy only. ABGA as determined by indirect immunofluorescence, ELISA, and western immunoblot were negative.

Abbreviations: ABGA, anti-basal ganglia antibodies; SC, Sydenham's chorea 


\section{DISCUSSION}

Both cases had recurrent or prolonged episodes of SC in childhood or early life. Extensive investigations for all other causes of chorea were either negative or normal. Neither patient had evidence of a recent streptococcal infection to suggest recurrent infection with a rheumatic strain of group A $\beta$-haemolytic streptococcus or fulfilled the modified Jones criteria for rheumatic fever. MRI showed no vascular or structural changes in the basal ganglia or thalami in either case. Exclusion of other causes of chorea coupled with a history of SC strongly suggests that these cases are due to late recurrences of SC.

The pathophysiological basis of late recurrences of SC has not been established. Acute SC is extremely closely associated with the presence of ABGA in the serum, $100 \%$ in a recent series. ${ }^{9}$ A study of intra-striatal infusion of purified ABGA into rat brain suggested that these antibodies might be pathogenic. ${ }^{10}$ Case studies of patients with SC treated with immunomodulating drugs such as intravenous immunoglobulin and steroids have reported an improvement in symptoms and provide evidence that these antibodies may be pathogenic. These data coupled with leucocyte infiltration pathologically ${ }^{6}$ and basal ganglia enlargement on imaging ${ }^{7}$ support an immune mediated pathology in SC.

Severe SC or multiple relapses could produce irreversible damage to the basal ganglia in some cases. The close association of ABGA with acute SC may suggest that immune mediated damage to the basal ganglia can occur. ABGA have been shown in persistent SC, but $30 \%$ of patients have no detectable antibodies. This may imply persistent chorea is due to basal ganglia damage in some patients rather than a reexacerbation of an immune response. ${ }^{9}$ Studies have also shown that even after recovery some SC patients retain hypersensitivity to dopaminergic agents and persistent oculomotor abnormalities have also been reported in $20 \%$ of patients who have recovered from SC. ${ }^{13}$

We suggest that although current evidence points to a central role for the immune system in the pathogenesis of acute and early relapses of SC, the absence of ABGA in patients with late recurrences suggests an alternative pathogenesis. The development of a co-existent pathology such as dementia or simply cumulative neuronal loss associated with ageing may be sufficient to produce dysfunction in previously damaged basal ganglia leading to the late re-emergence of chorea. This may have implications regarding the treatment of patients with multiple relapses of SC in early life to prevent senile chorea.

\section{Authors' affiliations}

A Church, G Giovannoni, Neuroimmunology Unit, Neuroinflammation, Institute of Neurology, Queen Square, London, UK

N A Harrison, Psychiatry, Institute of Psychiatry, De Crespigny Park, London, UK

P Rudge, Neurology, National Hospital of Neurology and

Neurosurgery, Queen Square, London, UK

A Nisbet, Neurology, Royal Sussex County Hospital, Brighton, UK

Competing interests: none declared

Correspondence to: $\operatorname{Dr} G$ Giovannoni, Neuroimmunology Unit, Neuroinflammation, Institute of Neurology, Queen Square, London, WCIN 3BG, UK; G.Giovannoni@ion.udl.ac.uk

Received 12 November 2003

In revised form 10 January 2004

Accepted 13 January 2004

\section{REFERENCES}

1 Cardoso F, Eduado C, Silva AP, et al. Chorea in fifty consecutive patients with rheumatic fever. Mov Disord 1997; 12(5):701-3.

2 Ayoub EM. Resurgence of rheumatic fever in the United States. The changing picture of a preventable illness. Postgrad Med 1992;92:133-4.

3 Aron AM, Freeman JM, Carter S. The natural history of Sydenham's chorea: review of the literature and long term evaluation on cardiac sequelae. Am J Med 1965;38:93-7.

4 Swedo SE, Leonard HL, Schapiro MB, et al. Sydenham's chorea: physical and psychological symptoms of St Vitus dance. Pediatrics 1993;91:706-13.

5 Mercadante MT, Busatto GF, Lombroso PJ, et al. The psychiatric symptoms of rheumatic fever. Am J Psychiatry 2000;157:2036-8.

6 Greenfield JG, Wolfsohn JM. The pathology of Sydenham's chorea. Lancet 1922;2:603-6.

7 Giedd JN, Rapoport JL, Kruesi MJP, et al. Sydenham's chorea: magnetic resonance imaging of the basal ganglia. Neurology 1995;45:2199-202.

8 Husby G, Van de Rijn I, Zabriske JB, et al. Antibodies reacting with cytoplasm of subthalamic and caudate nuclei neurones in chorea and acute rheumatic fever. J Exp Med 1976;144:1094-110.

9 Church AJ, Cardoso F, Dale R, et al. Anti-basal ganglia antibodies in acute and persistent Sydenham's chorea. Neurology 2002;59:227-31.

10 Halleft JJ, Harling-Berg CJ, Knopf PM, et al. Anti-striatal antibodies in Tourette syndrome cause neuronal dysfunction. J Neuroimmunol 2000;111:195-202. 